
Sur seleccionados en el XII Encuentro de Editores, La Paz, Bolivia, 2007, para ser publicados por los países integrantes durante el año 2008.

\title{
Corioamnionitis histológica en el recién nacido menor de 1000 gramos. Incidencia y resultados perinatales
}

\author{
MARIO MORAES C. ${ }^{1}$, MARÍA JOSÉ CANCELA², MARIZELL REPETTO ${ }^{3}$, \\ CARMEN GUTIÉRREZ4, VERÓNICA FIOL ${ }^{5}$, GABRIELA PIRIZ ${ }^{6}$, \\ EDGARDO CASTILLO ${ }^{7}$, ESTRELLA MEDINA ${ }^{8}$, MÓNICA LATTOF$^{2}$, RAÚL BUSTOS ${ }^{8}$ \\ 1. Profesor Adjunto, Departamento de Neonatología, Facultad de Medicina de la Universidad de la República, Uruguay \\ 2. Ex Asistentes de Departamento de Neonatología. Facultad de Medicina de la Universidad de la República, Uruguay \\ 3. Ex Profesora Adjunta de Pediatría, Facultad de Medicina de la Universidad de la República, Uruguay \\ 4. Directora del Servicio de Anatomía Patológica Pediátrica. Centro Hospitalario Pereira Rosell. Ministerio de Salud Pública \\ 5. Residente de Ginecología, Clínica Ginecotocológica "A", Facultad de Medicina de la Universidad de la República, Uruguay \\ 6. Asistente de Cátedra, Clínica Ginecotocológica " $C$ ", Facultad de Medicina de la Universidad de la República, Uruguay. \\ 7. Profesor Adjunto de Ginecología, Clínica Ginecotocológica "A", Facultad de Medicina de la Universidad de la República, \\ Uruguay. \\ 8. Profesor Departamento de Neonatología. Facultad de Medicina de la Universidad de la República, Uruguay.
}

\begin{abstract}
Histological chorioamnionitis in the newborn with birth weight less than $1000 \mathrm{~g}$ perinatal incidence and results

Objective: Determine the incidence of clinical and histological chorioamnionitis on extremely low birth weight infants and its correlation with perinatal outcome. Method: Descriptive and prospective study carried out between May 2004 and April 2005. All newborns weighting between $500-1000 \mathrm{~g}$ and with placental histopathological study were included. Results: A total of 52 infants fulfilled the inclusion criteria. Histological study of the placenta was performed in 44 patients (84.6\%). The average birth weight was $822.3 \mathrm{~g}$ (SD $127 \mathrm{~g}$ ) and the mean gestational age was 26 weeks (SD 2 weeks). Preterm labour was recorded in 28 mothers (63.6\%), where 18 (40.9\%) had premature membrane rupture. Histological diagnosis of chorioamnionitis was done in 27 cases $(61.4 \%)$, where $12(27.2 \%)$ had a clinical diagnosis of chorioamnionitis previously. In 13 of 27 cases with histological chorioamnionitis, no premature membrane rupture was recorded. $75 \%$ (9) of patients with clinical diagnosis presented chorioamnionitis with fetal response in the placenta at the histological study. In 5 cases, histological chorioamnionitis with fetal response was found in absence of clinical diagnosis. Early neonatal mortality was observed in 15 (34\%)
\end{abstract}

Este material fue presentado como póster en el Primer Congreso Uruguayo de Neonatología; Montevideo, 2005. Obtuvo el Premio Fundación Caldeyro Barcia.

Fecha recibido: 5 de julio de 2006

Fecha aprobado: 12 de setiembre de 2006

\section{Correspondencia:}

Dr. Mario Moraes

Presidente Batlle 2656. Montevideo, Uruguay.

E-mail:marmoraes@adinet.com.uy 
cases and mortality during hospitalization in 23 (52.2\%). Neonatal sepsis developed in 2 patients (4.5\%), but 11 cases (25\%) presented early clinical sepsis, all of them associated to histological chorioamnionitis $\left({ }^{*} \mathrm{p}=0.02\right)$. In 5 cases, necrotizing enterocolitis was observed $(11.3 \%)$ and intraventricular hemorrhage in $12(27.2 \%)$, in which 7 were grade 3 to 4 (16.2\%). Finally, 9 had bronchopulmonary dysplasia, associated to histological chorioamnionitis with fetal response $(* p=0.01)$. Conclusions: Histological chorioamnionitis has a high incidence; $44 \%$ of cases, it was possible to confirm that it preceded membrane rupture. Chorioamnionitis is often clinically silent and is diagnosed by the pathologist after birth. Clinical diagnosis of chorioamnionitis presents low sensitivity and high specificity in relation to histological chorioamnionitis. Clinical suspicion of ovular infection is highly suggestive of advanced infection with histological fetal response. Histological choriamnionitis is statistically associated to preterm labour and early clinical sepsis. Histological chorioamnionitis with fetal response is statistically associated with bronchopulmonary dysplasia and necrotizing enterocolitis.

(Key words: Chorioamnionitis, newborn, very low birth weight).

Rev Arch Pediatr Urug 2006; 77(3): 244-249

\section{RESUMEN}

Objetivo: Evaluar la incidencia de corioamnionitis clínica e histológica en los neonatos menores de 1000 gramos y su correlación con los resultados perinatales. Sujetos y Método: Se realizó un estudio descriptivo prospectivo entre mayo de 2004 y abril de 2005. Se incluyen recién nacidos con un peso al nacer entre 500 y 1000 g con estudio histopatológico de la placenta. Resultados: En el período analizado nacieron 52 menores de 1000 g, se realizó estudio de la placenta en 44 (84,6\%). El peso al nacer promedio fue de 822,3 g (DE 127 g); la edad gestacional promedio 26 semanas (DE 2 semanas). Presentaron trabajo de parto pretérmino 28 madres $(63,6 \%)$ y rotura prematura de membranas 18 (40,9\%). Se observó corioamnionitis histológica en 27 casos (61,4\%); 12 pacientes (27,2\%) presentaban corioamnionitis clínica. En 13 de los 27 casos de corioamnionitis histológica no se consignó ruptura de membranas ovulares. $75 \%$ de los casos con clínica de coriamnionitis presentaron, en el estudio histopatológico, corioamnionitis con respuesta fetal. En cinco casos se constató corioamnionitis histológica con respuesta fetal en ausencia de diagnóstico clínico. Se produjo muerte neonatal temprana en 15 (34,0\%) y durante la internación en 23 (52,2\%). Dos pacientes presentaron sepsis neonatal temprana (4,5\%), y 11 sepsis clínica temprana (25,0\%) que se asoció a corioamnionitis histológica $(* \mathrm{p}=0,02)$. Cinco pacientes presentaron enterocolitis necrotizante $(11,3 \%)$. Se observó hemorragia intraventricular en 12 (27,2\%), siete grados 3-4 (16,2\%). En nueve pacientes se comprobó displasia broncopulmonar, que se asoció a corioamnionitis histológica con compromiso fetal $\left({ }^{*} p=0,01\right)$. Conclusiones: La corioamnionitis histológica tiene elevada incidencia en la población estudiada, y en $44 \%$ de los casos se pudo comprobar que precede a la rotura de membranas. El diagnóstico clínico de corioamnionitis se correlaciona con la lesión histológica con baja sensibilidad y elevada especificidad. Se trata de una lesión que con mayor frecuencia es clínicamente silenciosa y que se diagnostica por el patólogo luego del nacimiento. La sospecha clínica de infección ovular es altamente sugestiva de lesión histológica avanzada con respuesta fetal. La corioamnionitis histológica se asocia a trabajo de parto pretérmino y sepsis clínica temprana. Cuando hay respuesta fetal histológica en el estudio de la placenta, se asocia a broncodisplasia pulmonar y enterocolitis necrotizante.

(Palabras clave: Corioamnionitis, recién nacido, recién nacido de muy bajo peso).

Rev Arch Pediatr Urug 2006; 77(3): 244-249

\section{Introducción}

El parto prematuro (PP) es un problema para el sistema asistencial por los elevados recursos que su correcta atención requiere y por la vinculación que tiene con la mortalidad neonatal, así como diferentes secuelas ${ }^{1,2}$. En el
Centro Hospitalario Pereira Rossell (CHPR) en el período 2001-2004 hubo 34.371 nacimientos, de los cuales 280 correspondieron a neonatos menores de 1000 g (0,8\% del total). Este grupo de recién nacidos presentó una tasa de mortalidad neonatal temprana de 217,9\%.

Es conocida la vinculación existente entre la 
infección ascendente y el PP. Se define como corioamnionitis clínica (CAC) al síndrome clínico de infección intrauterina caracterizado por fiebre mayor a $37,8^{\circ}$, sin otro foco aparente, asociado a amniorrea purulenta, contracciones uterinas, dolor uterino, taquicardia materna o fetal y leucocitosis.

Se define como corioamnionitis histológi$c a(\mathrm{CAH})$ al conjunto de cambios inflamatorios de la placenta, el cordón y las membranas ovulares que ocurren en respuesta a la invasión del líquido amniótico por microorganismos procedentes del canal del parto. Se caracteriza por el hallazgo de leucocitos polimorfonucleares de origen materno en las membranas ovulares y en la placa corial. Se reconoce como respuesta fetal histológica al hallazgo de migración de polimorfonucleares en las paredes de los vasos fetales de la placa corial y del cordón umbilical en dirección al amnios ${ }^{3,4}$.

Si bien la corioamnionitis ha sido considerada clásicamente como una infección que afecta fundamentalmente a la madre, se sabe que puede involucrar al feto. Se asocia a PP y morbimortalidad materna y fetal. Existe relación directa entre CAH y resultados neonatales $\operatorname{adversos}^{5-7}$.

\section{Sujetos y Método}

Se realizó un estudio prospectivo observacional de la población gestante que se asistió en el Hospital de la mujer del CHPR en el período comprendido entre mayo de 2004 y abril de 2005.

Se seleccionaron aquellos recién nacidos vivos con un peso al nacer entre 500 y 1000 gramos cuya placenta se envía para análisis histopatológico.

Se excluyeron los recién nacidos con malformaciones incompatibles con la vida, en los que sólo se realizaron medidas compasivas. Se excluyeron todos los casos de muertes fetales.

Se recolectaron los datos de forma prospectiva y se volcaron a una ficha protocolizada. Se analizaron las siguientes variables: ficha patronímica materna, patología durante el embarazo, conducta obstétrica y resultados perinatales.
De los datos patronímicos de la paciente se consideraron edad y patologías propias o asociadas a la gestación y de la conducta obstétrica se evaluó la forma de finalización de la gravidez.

De los resultados perinatales se consideraron mortalidad neonatal durante la internación, sepsis neonatal, sepsis clínica, leucomalacia periventricular, hemorragia intraventricular, enterocolitis necrotizante y retinopatía del prematuro.

El diagnóstico de corioamnionitis clínica fue realizado por el equipo médico actuante en cada caso. Se realizó en presencia de fiebre materna (37,8 ${ }^{\circ} \mathrm{C}$ o más) y dos o más de los siguientes elementos: taquicardia materna o fetal, dolor a la palpación uterina, irritabilidad uterina, fetidez en el líquido amniótico y leucocitosis en ascenso. Las placentas fueron analizadas en el Laboratorio de Patología Pediátrica del CHPR con protocolo uniforme que incluyó examen macroscópico minucioso, estudio histológico obligatorio de tres fragmentos de la placenta que incluyeron placa corial y basal, un fragmento de rollo de membranas y dos fragmentos del cordón umbilical, además de la toma de fragmentos de todas las áreas que tienen alteración macroscópica. La fijación del material se hizo con formol al 10\%. La inclusión se hizo en parafina y la coloración de rutina empleada fue la hematoxilina y eosina.

Se define como CAH a la presencia de infiltrado de PMN difuso o parcelar en el tejido conectivo del corion y/o el amnios. La respuesta fetal se mide por la migración de PMN a través de las paredes vasculares del cordón y/o de la placa corial ${ }^{8}$.

Los recién nacidos son valorados mediante dos hemogramas y la determinación de la proteína C reactiva en dos oportunidades, separados por un tiempo de 24 horas. Se realizaron dos hemocultivos por punción. Se realizó ecografía transfontanelar en las primeras 72 horas y luego con intervalo quincenal a mensual hasta el alta, se clasificaron las hemorragias intracraneanas según Papile.

El estudio de retinopatía a cargo de oftalmólogo especializado se realizó desde el primer mes de vida, con una periodicidad quincenal o con un intervalo menor según la evolución de cada paciente hasta el momento del alta. 
Se consideró sepsis neonatal temprana confirmada al crecimiento microbiológico en sangre, líquido cefalorraquídeo u orina extraídos en las primeras 72 horas de vida y sepsis clínica temprana, al diagnóstico realizado por neonatólogo actuante en el mismo período en presencia de alteraciones en el hemograma compatibles.

Se consideró displasia broncopulmonar a la necesidad de oxígeno suplementario luego de los 28 días de vida y una radiografía de tórax compatible con este diagnóstico.

El diagnóstico de enterocolitis se basa en signos clínicos más la presencia de neumatosis intestinal en la radiografía de abdomen y en el estudio de necropsia previamente autorizada.

\section{Resultados}

En el período analizado nacieron 52 recién nacidos menores de 1000 gramos que cumplían los criterios de inclusión, se realizó estudio de la placenta en 44 (84,6\%).

El peso al nacer promedio fue de 822,3 g (DE $127 \mathrm{~g}$ ) y la edad gestacional promedio de 26 semanas (DE 2 semanas).

Los datos maternos y del embarazo se presentan en la tabla 1. El promedio de la edad materna fue de 24 años \pm 6 . En un 43,1\% de la población gestante analizada se consignó el consumo de tabaco.

Las patologías maternas más prevalentes fueron la rotura prematura de membranas, con una frecuencia de 40,9\% ( $\mathrm{n}=18)$ y la amenaza de parto pretérmino 40,9\% ( $\mathrm{n}=18)$. Se observó un $27,2 \%(n=12)$ de CAC y $25,0 \%(n=11)$ de infecciones genitales bajas.

El 40,9\% $(n=18)$ finalizó su embarazo mediante un parto vaginal y el 51,9\% $(\mathrm{n}=26)$ terminó en cesárea; 73\% $(n=19)$ de ellas fueron de urgencia.

En 27 de 44 placentas analizadas se consignó el diagnóstico de CAH (61,3\%). En la tabla 2 se señalan las características de la muestra analizada en relación al diagnóstico histológico de corioamnionitis.

Se observó un incremento de rotura prematura de membranas, rotura prematura y prolongada de membranas e infecciones genitales bajas en el grupo de CAH que no es estadísticamente significativo.

Tabla 1. Características de la población $(n=44)$

\begin{tabular}{lrr}
\hline & n & $\%$ \\
\hline Edad materna (años \pm DE) & $24 \pm 6$ & \\
Fumadora & 19 & 43,1 \\
Hipertensión crónica & 3 & 6,8 \\
Ruptura prematura de membranas & 18 & 40,9 \\
Amenaza de parto de pretérmino & 18 & 40,9 \\
Desprendimiento previo de placenta & & \\
normoinserta (diagnóstico clínico) & 9 & 20,4 \\
Preeclampsia & 8 & 18,1 \\
Infección genital baja & 11 & 25,0 \\
Corioamnionitis clínica & 12 & 27,2 \\
\hline
\end{tabular}

Tabla 2. Características de la población según el diagnóstico de corioamnionitis histológica

\begin{tabular}{|c|c|c|c|c|}
\hline & \multicolumn{2}{|c|}{$\begin{array}{l}\text { Con CAH } \\
n=27\end{array}$} & \multicolumn{2}{|c|}{$\begin{array}{l}\text { Sin CAH } \\
n=17\end{array}$} \\
\hline & $\mathbf{n}$ & $\%$ & $\mathbf{n}$ & $\%$ \\
\hline Ruptura prematura de membrana & 14 & 51,8 & 4 & 23,5 \\
\hline Ruptura prematura prolongada de membrana & 10 & 37,0 & 4 & 23,5 \\
\hline $\begin{array}{l}\text { Desprendimiento previo de placenta normoinserta } \\
\text { (diagnóstico clínico) }\end{array}$ & 6 & 22,2 & 3 & 20 \\
\hline Preeclampsia & 0 & 0 & $\begin{array}{c}8 \\
\text { p } 0,0001\end{array}$ & 47 \\
\hline Corioamnionitis clínica & 9 & 33,3 & 3 & 17,6 \\
\hline Trabajo de parto & 22 & 81,4 & $\begin{array}{ll}6 \\
p & 0,01\end{array}$ & 35,2 \\
\hline
\end{tabular}

$\mathrm{CAH}$ : corioamnionitis histológica 
En 13 de los 27 casos de CAH no se consignó ruptura de las membranas ovulares.

Se inició trabajo de parto pretérmino en forma espontánea en el 81,4\% de las pacientes portadoras de $\mathrm{CAH}$.

El 75\% (9 de los 12 casos) de las CAC presentaron $\mathrm{CAH}$ con respuesta fetal en la investigación de la placenta. En cinco casos se realizó diagnóstico de CAH con respuesta fetal en ausencia de diagnóstico clínico de corioamnionitis.

Los resultados neonatales se presentan en las tablas 3 y 4.
Se constató 34,0\% de muerte neonatal temprana $(\mathrm{n}=15)$ y un $52,2 \%(\mathrm{n}=23)$ de mortalidad neonatal durante la internación.

De la morbilidad neonatal se consignó el diagnóstico de sepsis clínica en las primeras 72 horas en el $25,0 \%(n=11)$ y 4,5\% $(n=2)$ sepsis neonatal temprana.

Se constató hemorragia intraventricular en el 27,2\% ( $n=12)$ de los neonatos; en 7 de los 12 casos $(58,3 \%)$ correspondieron a grados 3-4.

El 20,4\% ( $=9)$ presentó displasia broncopulmonar. Se observó un 11,3\% $(n=5)$ de enterocolitis necrotizante.

Tabla 3. Resultados neonatales y relación con presencia de corioamnionitis histológica

\begin{tabular}{|c|c|c|c|c|}
\hline & \multicolumn{2}{|c|}{$\begin{array}{c}\text { Con CAH } \\
n=27\end{array}$} & \multicolumn{2}{|c|}{$\begin{array}{c}\text { Sin CAH } \\
n=17\end{array}$} \\
\hline & $\mathbf{n}$ & $\%$ & $\mathbf{n}$ & $\%$ \\
\hline Muerte & 15 & 55,5 & 8 & 47,0 \\
\hline Muerte neonatal temprana & 9 & 33,3 & 6 & 35,2 \\
\hline Retinopatía & 10 & 37,0 & 5 & 29,4 \\
\hline Hemorragia intracraneana & 10 & 37,0 & $\left(p: \frac{2}{0,06)}\right.$ & 11,7 \\
\hline Leucomalacia & 1 & 2,2 & 0 & 0 \\
\hline Enterocolitis necrotizante & 5 & 18,5 & 0 & 0 \\
\hline Displasia broncopulmonar & 8 & 29,6 & 1 & 5,8 \\
\hline Sepsis clínica temprana & 10 & 37,0 & $\begin{array}{c}1 \\
\left({ }^{*} p: 0,02\right)\end{array}$ & 5,8 \\
\hline
\end{tabular}

$\mathrm{CAH}$ : corioamnionitis histológica

Tabla 4. Resultados neonatales y corioamnionitis histológica con compromiso fetal

\begin{tabular}{|c|c|c|c|c|}
\hline & \multicolumn{2}{|c|}{$\begin{array}{l}\text { CAH con respuesta fetal } \\
\qquad n=14\end{array}$} & \multicolumn{2}{|c|}{$\begin{array}{l}\text { CAH sin respuesta fetal } \\
\qquad n=30\end{array}$} \\
\hline & $\mathbf{n}$ & $\%$ & $\mathbf{n}$ & $\%$ \\
\hline Muerte & 7 & 50 & 16 & 53,3 \\
\hline Muerte neonatal temprana & 4 & 28,5 & 11 & 36,6 \\
\hline Retinopatía grados 2-3 & 1 & 7,14 & 4 & 13,3 \\
\hline Hemorragia intracraneana & 2 & 14,2 & 10 & 33,3 \\
\hline Leucomalacia & 1 & 7,14 & 0 & 0 \\
\hline Sepsis clínica & 5 & 35,7 & 6 & 20,0 \\
\hline Enterocolitis & 4 & 28,5 & $\begin{array}{c}1 \\
* p: 0,01\end{array}$ & 3,3 \\
\hline Displasia broncopulmonar & 6 & 42,8 & ${ }^{*} p:{ }^{3} 0,01$ & 10,0 \\
\hline
\end{tabular}

CAH: corioamnionitis histológica 


\section{Discusión}

Este artículo reporta una incidencia de corioamnionitis histológica de $61,3 \%$ en prematuros menores de $1000 \mathrm{~g}$. Otros estudios publicados han reportado una incidencia de corioamninitis en el nacimiento de un niño menor de 1000 gramos entre $31 \%$ y $83 \%{ }^{9}$.

Se observó alta incidencia de corioamnionitis histológica con respuesta fetal en la población analizada. De las 12 pacientes en las que se planteó el diagnóstico clínico de corioamnionitis, en nueve casos se confirmó por estudio de la placenta la presencia de respuesta fetal. La sospecha clínica de corioamnionitis es altamente sugestiva de compromiso fetal. En cinco casos se realizó diagnóstico de corioamnionitis histológica con respuesta fetal en ausencia de sospecha clínica, estos elementos están a favor de que la corioamnionitis es una infección materno-fetal desde su inicio.

Se demuestra en este trabajo que el diagnóstico clínico de corioamnionitis presenta una baja sensibilidad (33,3\%) y una especificidad elevada $(82,3 \%)$ para la presencia de corioamnionitis histológica. De Felice ${ }^{10}$ informa que la evidencia clínica de corioamnionitis identifica el compromiso histológico con una sensibilidad de $24 \%$ y una especificidad de $87 \%$ en el $81,4 \%$ de las pacientes con diagnóstico histológico de corioamnionitis el nacimiento es precedido por trabajo de parto, mientras que esto sólo ocurre en 23,5\% de las pacientes sin corioamnionitis histológica. Esta observación concuerda con la idea de que el inicio del trabajo de parto pretérmino se asocia con corioamnionitis ${ }^{11,12}$.

El 48,2\% de la corioamnionitis histológica no presenta rotura prematura de membranas. Existen investigaciones que han documentado la presencia de corioamnionitis, semanas o meses previos a la rotura de membrana o trabajo de parto pretérmino. Se puede producir infección subclínica antes de la rotura de membranas; ser la causa y no la consecuencia de esta complicación ${ }^{13-15}$.

En el 18,1\% de las pacientes se realizó cesárea por preeclampsia y no se encuentra corioamnionitis histológica en el estudio de la placenta.
La presencia de preeclampsia se asocia en forma estadísticamente significativa a ausencia de coriamnionitis histológica (*p 0,0001). La presencia de corioamnionitis histológica se asocia a la presencia de sepsis clínica con valor estadístico.

En todos los pacientes se realizó administración de antibiótico en el caso de rotura de membranas de pretérmino o trabajo de parto pretérmino. Existe consenso que la utilización de antibióticos en el caso de rotura pretérmino de membranas disminuye el riesgo de sepsis neonatal y prolonga la gestación ${ }^{16}$. La incidencia de sepsis neonatal temprana es coincidente con el 5\% al 8\% informado en otros estudios ${ }^{17}$. Se constató el diagnóstico de sepsis clínica en 25,0\% de los recién nacidos. La utilización de antibióticos dificulta la valoración de signos clínicos de sepsis y aumenta la incidencia de bacterias resistentes o poco habituales ${ }^{16,17}$.

La presencia de CAH se asocia a trabajo de parto pretérmino. La CAH con compromiso fetal se asocia en forma estadísticamente significativa con enterocolitis necrotizante $\left({ }^{*} \mathrm{p}: 0,01\right)$ y displasia broncopulmonar (*p: 0,01).

Otros autores informan una mayor incidencia de resultados neonatales adversos en presencia de corioamnionitis con compromiso fetal, como ser retinopatía grados 2-3, hemorragias intracraneanas en general y en grados 3-4, leucomalacia periventricular y broncodisplasia pulmonar-7,18-20. Otro estudio realizado en nuestro medio en 413 placentas prematuras del CHPR mostró que globalmente analizadas el $59 \%$ tenían corioamnionitis, pero que en los productos menores de $800 \mathrm{~g}$ el porcentaje con CAH fue de $72 \%{ }^{21}$.

\section{Conclusiones}

La corioamnionitis histológica tiene elevada incidencia en los recién nacidos menores de 1000 g y en $44 \%$ de los casos se pudo comprobar que precede a la rotura de membranas. El diagnóstico clínico de corioamnionitis identifica CAH con baja sensibilidad y elevada especificidad. La sospecha clínica de infección ovular es altamente sugestiva de compromiso fetal. $\mathrm{La}$ CAH se asocia en forma estadísticamente 
significativa a trabajo de parto pretérmino y sepsis clínica temprana. La CAH con compromiso fetal en la placenta se asocia en forma estadísticamente significativa a broncodisplasia pulmonar y enterocolitis necrotizante.

\section{Referencias}

1.- Russell P: Inflammatory lesions of the human placenta. Clinical significance of acute chorioamninitis. Am J Diag Gynecol Obstet 1979; 1: 127-37.

2.- Redline DW, O Riordan A: Placental lesión associated with cerebral palsy and neurologic impairment following term birth. Arch Pathol Lab Med 2000; 124: 785-91.

3.- Asraut T: Infección intraamniótica en pacientes con rotura de membranas pretérmino antes del trabajo de parto. Fisiología, detección y tratamiento. Clin Perinatol 2001; 4: 701-16.

4.- Hillier SL, Martius J, Krohn M, et al: A case control study of chorioamnionitis infection and histologic chorioamnionitis in prematurity. N Engl J Med 1988; 319: 972-78.

5.- $W u$ YW, Coldford Jr JM: Choroamnionitis as a risk factor for cerebral palsy. JAMA 2000; 284: 1417-24.

6.- Wilson-Costello D, Borawski E, Friedman H, Redline $R$, Fanaroff $A A$, Hack $M B$, et al: Perinatal correlates of cerebral palsy and other neurologic impairment among very low birth weight children. Pediatrics 1998; 102: 315-22.

7.- Willoughby RE, Nelson KB: Corioamnionitis y lesión encefálica. Clin Perinatol 2002; 4: 589-607.

8.- Redline R, Faye-Petersen O, Heller D, Quresi F, Savell $V$, Vogler $C$ : Amniotic infection syndrome: Nosology and reproducibility of placental reaction patterns. Pediatr Dev Patol 2003; 6: 435-48.

9.- Goldemberg RL, Hauth JC, Andrews WW: Mechanisms of disease: intrauterine infection and preterm delivery. N Engl J Med 2000; 342: 1500-7.

10.- De Felice $C$, Toti P, Laurini R: Early neonatal brain injury in histologic chorioamnionitis. J Pediatr 2001; 138: 101-4.

11.- Guzick DS, Winn K: The association of chorioamnionitis with preterm delivery. Obstet Gynecol 1985; 65: 11-6.

12.- Arias F, Rodríguez L, Rayne SC: Maternal placental vasculopathy and infection: two distinct subgroups among patients with preterm labor and preterm rupture membranes. Am J Obstet Gynecol 1993; 168: 585.

13.- Romero $R$, Quintero $R$, Oyarzum E: Intraamniotic infection and the onset of labor in preterm premature rupture of the membranes. Am J Obstet Gynecol 1988; 159: 661.

14.- Goldemberg RL, Culhane JF: La infección como causa de parto pretérmino. Clin Perinatol 2003(4): 667-90.

15.- Parry S, Strauss JF III: Premature rupture of the fetal membranes. N Engl J Med 1998; 338: 663-70.

16.- Maymon E, Chaim W, Sheiner E: A Review of ramdomized clinical trial of antibiotic therapy in preterm premature rupture of the membranes. Arch Gynecol Obstet 1998; 261: 173-81.

17.- Mercer BM, Miodovnik M, Thurnau GR, Goldenberg $R L$, Das AF, Ramsey $R D$, et al: Antibiotic therapy for reduction of infant morbidity alter preterm premature rupture of the membranes. A ramdomized controlled trail. National Institute of child Health and Human Development Maternal-Fetal Medicine Units Network. JAMA 1997; 278: 989-95.

18.- Shalak LF, Laptook AR, Jafri HS, Ramilo O, Perlman $J M$ : Clinical chorioamnionitis, elevated cytokines, and brain injury in term infants. Pediatrics 2002; 110: 673-80.

19.- Hitti J, Tarczy-Hornoch P, Murphy J: Amniotic fluid infection, cytokines, and adverse outcome among infants at 34 weeks' or less. Obstet Gynecol 2001; 98: 1080-8.

20.- Watterberg KL, Demers LM, Scott SM, Murphy S: Chorioamnionitis and early lung inflammation in infants in whom bronchopulmonary dysplasia develops. Pediatrics 1996; 97: 211-5.

21.- Beltramo P, Gutiérrez C: Corioamnionitis histológica y parto prematuro en el Centro Hospitalario Pereira Rossell. Arch Ginecol Obstetr (en prensa). 